\title{
Interaction between main line standing waves and side branch resonance: stochastic signal attributes
}

\author{
M. Shehane, E. Moore \& A. Ruggles \\ University of Tennessee, Department of Nuclear Engineering, Knoxville, \\ USA
}

\begin{abstract}
Acoustic standing waves may be established in main gas delivery lines that are caused by resonance in branch lines. Conventional theory predicts branch line resonance frequency from the geometry of the branch and main line diameter. Data are presented that show the branch line standing wave couples with the main line standing wave, and a range of side branch resonance amplitude and frequency values are possible. Further, the resonance amplitude varies over long periods, and data acquisition using a short moving time window is employed to examine the Power Spectral Density (PSD) as a function of time. Peak amplitude values from the moving time window PSDs are identified and displayed in a Probability Density Function (PDF). The combined PSD and PDF information allows repeatable system characterization as a function of operating parameters and geometry, and data of this type are presented from a low-pressure air test facility.
\end{abstract}

Keywords: branch line resonance, acoustics, standing waves, fluid-structure interaction.

\section{Introduction}

Small branch lines off larger main delivery lines can exhibit acoustic resonance. Branch lines with a reflective obstruction, such as a valve or instrument, are susceptible to a $1 / 4$ wavelength standing wave, with a pressure node positioned near where the branch meets the main line, and a pressure anti-node positioned at the obstruction. Of course, higher modes are possible with an odd number of quarter wavelengths existing in the branch such that, 


$$
f_{\text {natural }}=\frac{n c}{4 L} \quad n=1,3,5,7 \ldots .
$$

where $\mathrm{c}$ is the sound speed and the characteristic length, L, is the branch length. The branch length is sometimes extended by some fraction of the diameter.

The most common drive for resonance in the branch line is vortex shedding at the branch, where vorticity in the fluid near the wall of the main line becomes free at the branch, and diverts flow periodically into the downstream branch wall, creating periodic pressure perturbations that serve as the energy source for the standing wave in the branch, Rockwell and Naudacher [1]. The frequency of pressure pulses created by the vortex shedding is predicted using the Strouhal number, $\mathrm{St}=\mathrm{fd} / \mathrm{v}$, with the branch diameter providing the length scale, and $\mathrm{v}$ set to the flow velocity in the main line. Coupling between the branch acoustic response and the vortex shedding behaviour allow for a branch to resonate for values for Strouhal number ranging roughly from 0.2 to 0.6 , Ziada and Shine, [2]. Strouhal number near 0.4 normally gives the largest amplitude acoustic response in the branch. These values vary with system parameters such as Reynolds number, as examined by Ziada and Buhlmann [3] Bruggeman [4] and Kriesels et al [5].

While the basic theory provides design guidance associated with the resonance in the branch, the excitation of standing waves in the main line has received little attention. A few studies examine acoustic coupling of branch line modes through the main line, Coffman and Bernstein [6], Ziada and Buhlmann [3], and Ziada and Shine [2]. However, these studies remain concerned with the loading of components in the branch line, such as safety relief valves.

Past modelling efforts have focused on the energy budget of the branch line only, with the vortex shedding mechanism as the energy source, and the energy sinks including damping mechanisms in the branch and acoustic radiation into the main line, Bruggeman [4] and Kriesels et al [5]. In circumstances where damping is low, and outlet paths for the acoustic energy in the main line are limited, the amplitude of the standing wave pattern in the main line can grow quite large, especially if multiple branches are present. Pressure variations in the main line at the branch position influence the acoustic performance of the branch and can modify the branch resonant frequency and amplitude. This paper presents data showing influence of a standing wave in the main line on the side branch pressure amplitude. A previous study using the same facility examined the frequency variation [7]. The side branch resonance amplitude varies over long times, making characterization of the system behaviour impossible unless attributes of the signal variability are included. A Power Spectral Density (PSD) is developed for a short moving time window. The short window is moved over a long time history, and a PSD is developed for each short window. The peak PSD amplitude of the side branch resonance is identified for each window, and the peak amplitude values from all the short windows are presented in the form of a Probability Density Function (PDF) for the long time history. An average PSD is evaluated by adding PSD outcomes for all windows, and dividing by the total number of window evaluations. The peak amplitude of the average PSD, 
and the variation in PSD peak amplitude values from the short time windows, depend on branch position relative to the standing wave pattern in the main line.

\section{Wave forms in main lines and how they interface with branch positions}

Standing waves of one-quarter wavelength may be induced in closed branch lines as represented in eq. 1 for the primary mode with $n$ equal unity. The model assumes that the ratio of the diameter of the branch line over the diameter of the main line is small, such that the pressure at the branch line entrance is near zero. In the case where standing pressure waves build in the main line, the pressure at the inlet of the branch line is equal to the pressure in the main line at the location of the branch. This will influence the effective wavelength and frequency of the branch, and also will influence the net amount of acoustic energy the branch can broadcast into the main line.

\section{Experimental}

This study examines the influence of the side branch position on the side branch resonance amplitude and amplitude variability. An atmospheric pressure, air test facility capable of Mach 0.14 flow is used to examine basic attributes of the relationship between standing waves in the main line and a single branch line. The main line is $7.6 \mathrm{~cm}$ in diameter with length $244 \mathrm{~cm}$. A single branch of diameter $1.9 \mathrm{~cm}$ and length $16.0 \mathrm{~cm}$ is attached to a pipe segment. The pipe segment with branch is moved along the main line. The pipe segment with branch contains 2 through wall mounted PCB Piezotronics ${ }^{\mathrm{TM}}$ microphones. A model 377B10 microphone is in the side branch, and model 377A12 microphone is in the main line at the branch position, both connected to Model 426B03 Preamps. Examination of acoustic pressures in the main line, and of the resonance amplitude in the branch exposed complex behaviour that was initially difficult to interpret. The data acquisition and data handling methods reported here were developed over approximately two years of effort. Details of the facility were presented in a previous paper [7].

\subsection{Matrix for data acquisition}

This experimental campaign examines system performance as the branch position is moved along a main line of constant length, with emphasis on attributes of the resonance amplitude. The main line length was set to $244 \mathrm{~cm}$, within $7 \mathrm{~mm}$, for every setup. The branch distance from the inlet was initially set to $61,71.1,81.3,90.8,102.9,111.8$, and $121.9 \mathrm{~cm}$. This was accomplished by using an array of $7.6 \mathrm{~cm}$ diameter Schedule 40 PVC piping segments with couplings. The position of the flow measurement instruments did not change between tests. Pressure data from the side branch exhibited a pattern that motivated further data acquisition to better capture minimum and maximum amplitude locations. 


\subsection{Data reduction and calculations}

The branch line used for reported data was $16.0 \mathrm{~cm}$ long. Therefore, with sound speed equal to $340 \mathrm{~m} / \mathrm{s}$ the expected frequency is $531 \mathrm{~Hz}$. Time histories were collected at 5,000 samples per second, with total time of 60 seconds constructed from six separate 10 second samples. A moving time window of width one second marches across the total time history in time increments of $1 / 2$ second. A PSD is evaluated for each of these windows, and the peak amplitude from the PSD is evaluated. The array of peak amplitude values thus created is displayed in a PDF histogram. A PSD created by averaging all individual window PSDs is also displayed for each measurement configuration.

\subsection{Results}

The peak amplitude data from the PSD created by averaging 119 PSDs from each 60 second time history is shown in fig. 1. A Probability Density Function (PDF) is created from the array of moving window peak PSD amplitude values. The PDF and the average of all moving window PSDs are provided for three specific measurement locations in fig. 2, fig. 3 and fig. 4 . Figure 2 corresponds to a peak amplitude location, fig. 3 is for a middle amplitude location, and fig. 4 corresponds to a minimum amplitude location. The locations corresponding to the data in fig. 2, fig. 3 and fig. 4 are denoted on fig. 1.

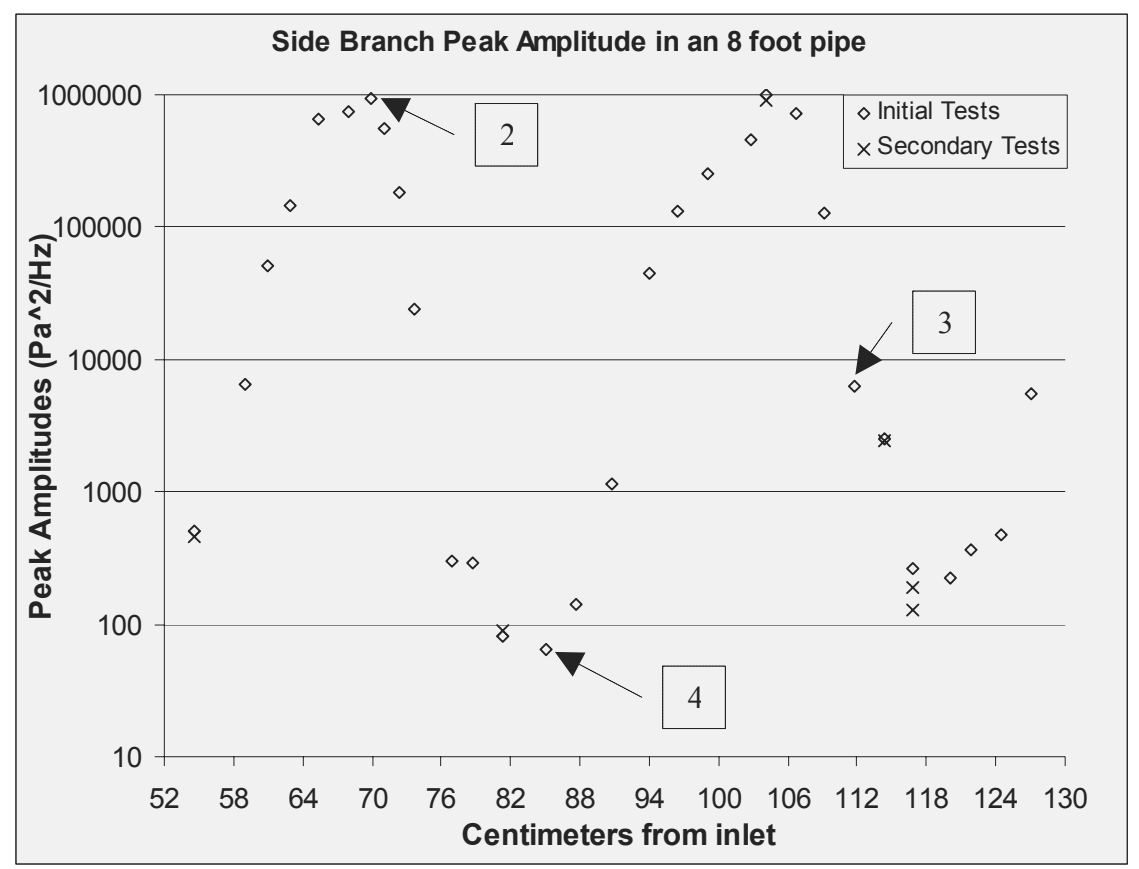

Figure 1: $\quad$ Averaged PSD amplitudes. 

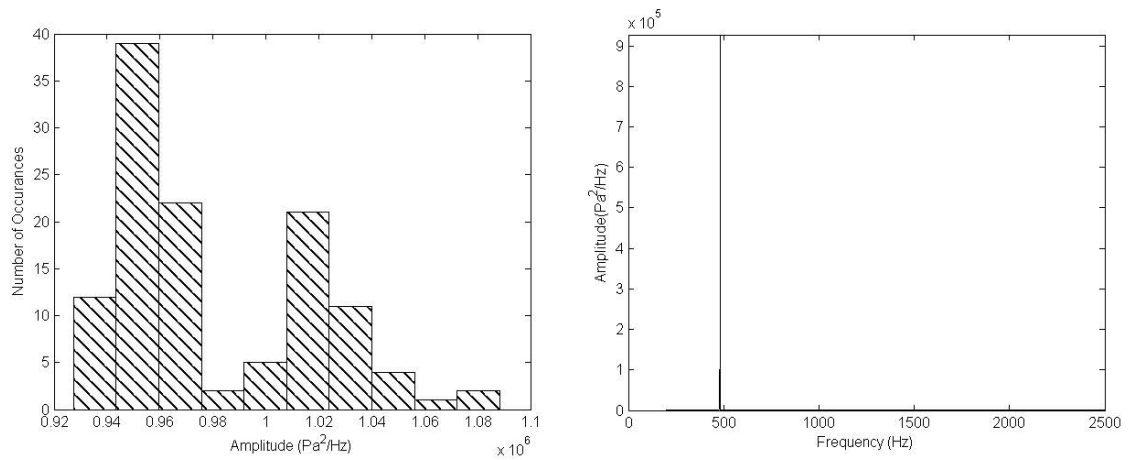

Figure 2: $\quad$ Amplitude PDF and PSD $69.9 \mathrm{~cm}$ from inlet.
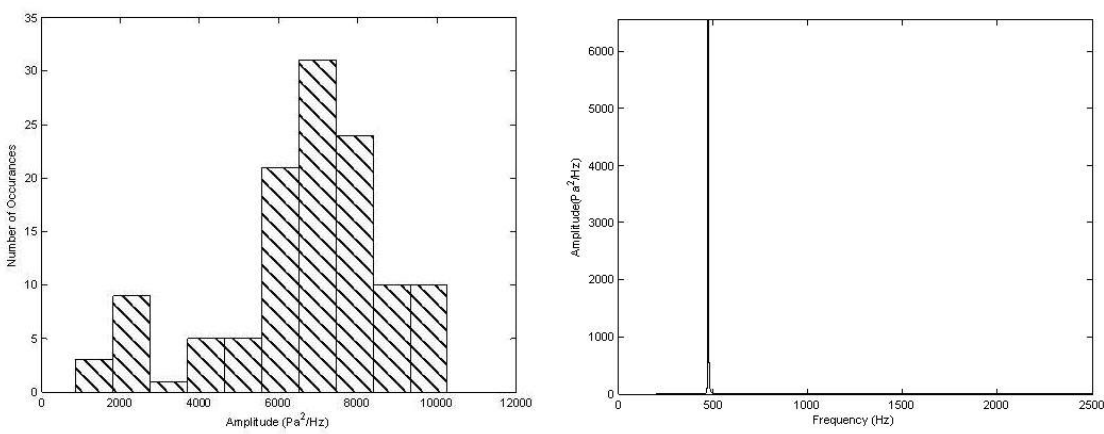

Figure 3: $\quad$ Amplitude PDF and PSD $112 \mathrm{~cm}$ from inlet.
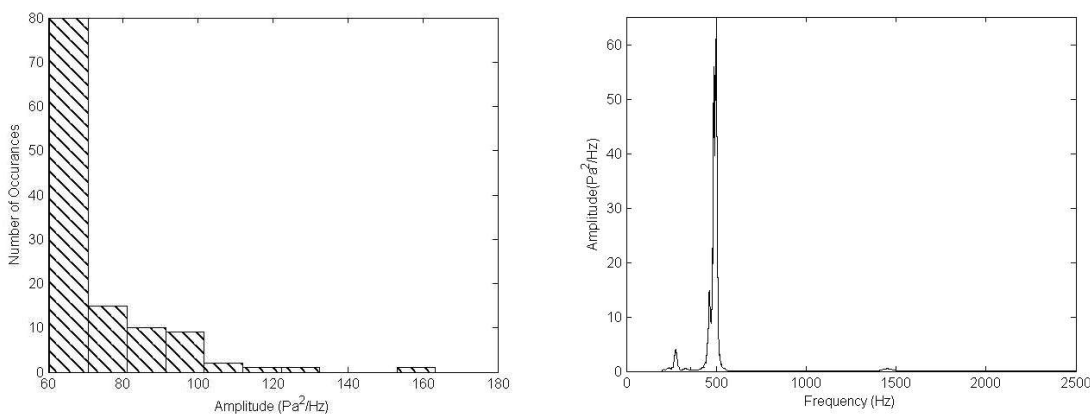

Figure 4: $\quad$ Amplitude PDF and PSD $85.1 \mathrm{~cm}$ from inlet.

\subsubsection{Data handling adequacy}

The waveform produced by the branch line can have a great deal of variance in amplitude, and the length of the time history required to characterize the behaviour must first be established. Figure 5 shows the average PSD for the 61 


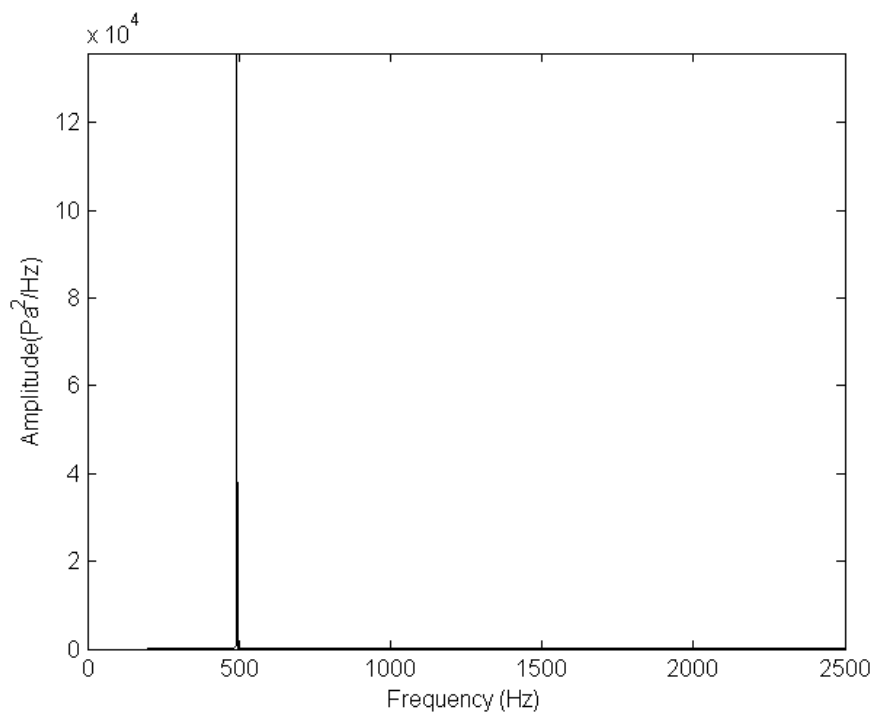

Figure 5: $\quad$ Averaged PSD for $61 \mathrm{~cm}$ position, one minute history.

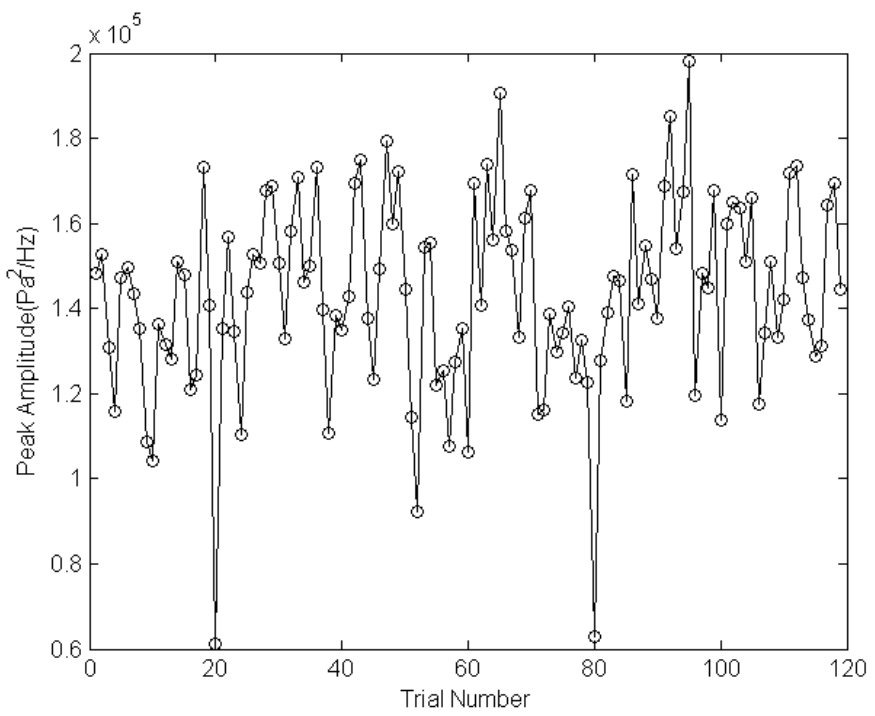

Figure 6: Window PSD peak amplitude values.

cm position, and fig. 6 shows the variability in the peak PSD amplitude at resonance. Figure 7 shows the variability in the running average peak amplitude value. This study shows the average value has become reasonably stable after 60 seconds, or 119 window evaluations. The variability in the amplitude is a 
function of the measurement location, so this outcome may not be universal. Therefore, the running average amplitude is updated with each time history to allow review of the adequacy of the history length. The 60 second history is adequate for all data presented here.

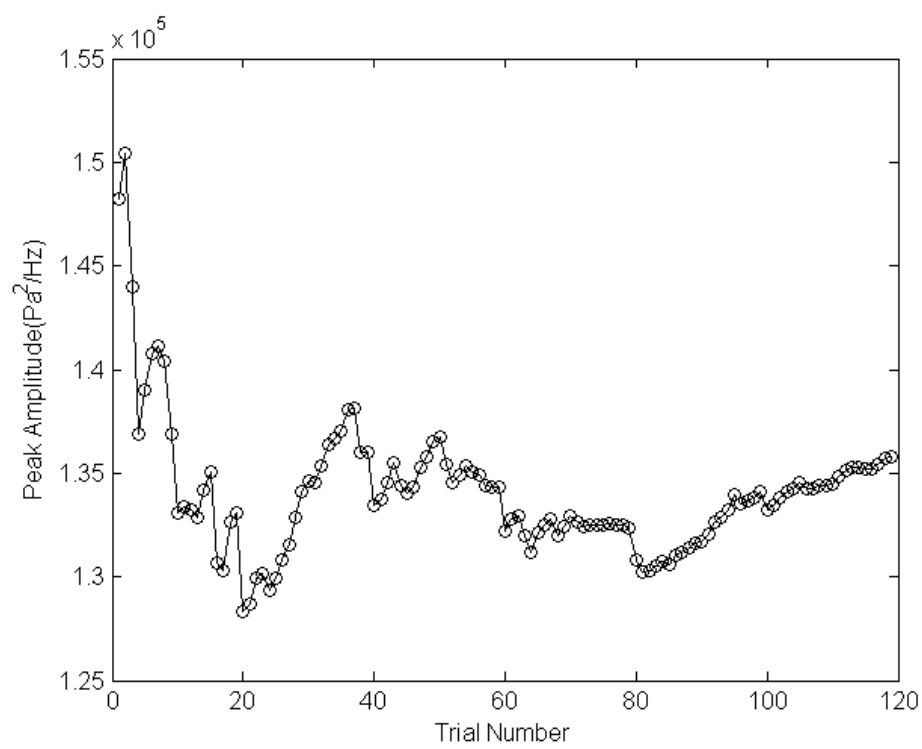

Figure 7: $\quad$ Running average of peak amplitude values.

A one second moving window width with $1 / 2$ second overlap is used throughout this evaluation. A two second window with one second overlap, and a $1 / 2$ second window with $1 / 4$ second overlap were examined before selecting these as appropriate and representative values. Only pressure data from the branch end are reported here, but data from the main line were also collected.

\section{Conclusions}

The focus on peak amplitude is related to the component load prediction and endurance prediction for design purposes. Fifty load cycles are required to achieve near full displacement and stress amplitudes for components with structural damping near $1 \%$. This implies that a pressure signal with amplitude variability should be evaluated using a moving window width that allows at least 50 cycles of the load frequency of interest if peak stress values are to be attained. This insures the peak amplitude relevant to predicting peak loading is discovered for that load frequency. If the loading is broad band, then a range of window widths should be explored to discover the range of pertinent peak load amplitudes. This example offers beginnings of a connection between a loading with stochastic attributes, and engineering design practice to achieve desired 
component endurance. There are parallels between the use of the PDF for peak amplitude at a specific resonance frequency, used here, and the use of the PDF to describe wind loadings for structures [8] and wave loadings for ships and off shore platforms.

Previous work shows branch resonance frequencies couple with main line resonance frequencies, allowing a branch to resonate over a range of frequencies as the length of the main line is altered. The peak amplitude of the resonance in the branch and main line is also a strong function of the branch position relative to the standing wave in the main line, and the variability in the peak amplitude is also a strong function of branch position. These realizations are important to engineering applications where the branch resonance causes dynamic loading of system components such as pipe supports, safety relief valves, steam dryers and steam super-heaters. Current engineering practice does not include consideration of the standing wave patterns in the main line when branch line resonance is evaluated.

\section{References}

[1] Rockwell, D. and Naudascher, E., "Review-Self Sustaining Oscillations of Flow Past Cavities." Journal of Fluids Engineering, Vol. 100, 152-165, 1978.

[2] Ziada, S. and Shine, S., "Strouhal Numbers of Flow-Excited Acoustic Resonance of Closed Side Branches." Journal of Fluids and Structures, Vol. 13, 127-142, 1999.

[3] Ziada, S. and Buhlmann, E. T., "Multiple Side-Branches as Tone Generators." Proceedings of International Mechanical Engineering Conference, paper 0416/009, pp. 435-444, 1991.

[4] Bruggeman, J. C., "Flow Induced Pulsations in Pipe Systems." PhD Thesis, Technische Universiteit Eindhoven, 1987.

[5] Kriesels, P. C., Peters, M. C. A. M., Hirschberg, A., Wijnands, A. P. J., Iafrati, A., Riccardi, G., Piva, R., Bruggeman, J. C., "High Amplitude Vortex Induced Pulsations in Gas Transport Systems." Journal of Sound and Vibration, Vol. 184, 343-368, 1995.

[6] Coffman, J. T. and Bernstein, M. D., “Failure of Safety Valves Due to Flow Induced Vibration.” Journal of Pressure Vessel Technology, Vol. 102, 112118, 1980.

[7] McKinnis, P., Miller, B, and Ruggles A. E., "Interaction Between Main Line Standing Waves and Side Branch Resonance Frequencies." Proceedings of WIT Fluid Structure Interaction and Moving Boundary Problems IV, pp. 337-349, The New Forest, UK, May 14-16, 2007.

[8] Blevins, R. D., "Flow Induced Vibration" $2^{\text {nd }}$ Edition, Krieger Publishing, 2001 . 